\title{
Le frontiere di Claudio Magris
}

\section{Ernestina Pellegrini}

Università di Firenze

\begin{abstract}
Questo saggio, che ha la forma eccentrica di appunti, presenta alcune riflessioni in margine al tema della frontiera nell'opera narrativa e saggistica di Claudio Magris. È strutturato in due parti: la prima consiste in un esame ravvicinato dell'ultima sua opera, $L a$ mostra, ritratto del pittore triestino Vito Timmel, la seconda, riunisce invece un elenco di citazioni sul motivo del confine estrapolate qua e là dalle opere dello scrittore.
\end{abstract}

Parole chiave: superuomini alla rovescia, mostra, dimenticare, disperazione esistenziale, spartiacque, Vito Timmel.

\begin{abstract}
This article, which is written eccentrically in note form, sets out a number of reflections on the theme of the frontier in the narratives and essays of Claudio Magris. The article is in two parts. The first of these is a close assessment of his latest work, La Mostra, a portrait of the Triestine painter Vito Timmel; the second, on the other hand, draws together a catalogue of quotations concerning the border motif, drawn here and there from the writer's work.
\end{abstract}

Key words: supermen turned inside out, mostra, forget, existential desperation, watershed, Vito Timmel.

Questo mio saggio, che ha la forma eccentrica di un insieme di appunti, vuole offrire alcune riflessioni in margine al tema della frontiera nell'opera narrativa e saggistica di Claudio Magris. È strutturato in due parti fortemente sbilanciate: la prima consiste in un esame ravvicinato dell'ultima opera dello scrittore, La mostra, edito da Garzanti nel 2000 (per un esame delle opere precedenti mi sia permesso rimandare il lettore alla monografia che ho scritto per l'editore di Bergamo Moretti\&Vitali nel 1996 col titolo Epica sull'acqua). La secon$\mathrm{da}$, che segue il movimento lirico-aforistico della prima come un'arida coda esemplificativa, vorrebbe offrire invece un semplice elenco di citazioni sul motivo del confine estrapolate qua e là dalle opere dello scrittore (mi sia permesso segnalare che su questo argomento è stata scritta una tesina da una giovane 
docente dell'Università di Scutari, Alma Hafizi, che ha potuto svolgere la ricerca sotto la mia guida con una borsa di studio del Ministero degli Esteri italiano. Il lavoro si intitola Claudio Magris: la morale che supera le frontiere; il lungo saggio è inedito ed è tuttora consultabile presso il Dipartimento di Italianistica dell'Università di Firenze).

Qualche anno fa è stato girato un film che è stato messo su videocassetta per Garzanti col titolo Fra il Danubio e il mare. Il mondo di Claudio Magris, per la regia di Francesco Conversano e Nene Grignaffini, prodotto da Movie Movie, con un'ampia intervista allo scrittore. Alla cassetta è unito un piccolo libro dove Claudio Magris parla dei luoghi, delle cose e delle persone da cui nascono i suoi libri, facendo così, in qualche modo, piazza pulita dei suoi critici. Insomma, oggi c'è un repertorio multimediale in cui si spiega Magris con Magris e noi critici si può anche fare le valige. Ma io le valige non le faccio, e allora per per questa occasione mi sono limitata a una lettura abbastanza approfondita della Mostra, l'ultimo testo di Magris. Non le faccio anche perché mi illudo di essere, per tutta una serie di motivi, una delle lettrici più congeniali di questo testo, che non è di per sé un testo facile. ${ }^{1} \mathrm{E}^{\prime}$ un minuscolo libro coinvolgente e sconvolgente, un libro bello di per sé ma spinoso, cioè pieno di spine. Voglio dire che è un libro cento volte più bello se si conosce anche qualcosa sulla vita e sull'opera di Vito Timmel, un pittore strampalato e geniale che è morto pazzo nel manicomio di Trieste, soprattutto se si è letto il suo Magico Taccuino, un romanzo-diario, che lui dettava oralmente ad Anita Pittoni, la quale lo avrebbe pubblicato tantissimi anni dopo, nel 1973, nelle eleganti edizioni dello "Zibaldone», con due introduzioni, una di Claudio Magris e l'altra di Franco e Franca Basaglia. ${ }^{2}$

Quando ci si trova fra le mani questo ultimo libro di Claudio Magris, $\mathrm{La}$ Mostra, di 65 pagine che si leggono tutte d'un fiato, ci si chiede: «Che cos è?». Un libro dove non si racconta nel vero senso della parola, perché nel testo non c'è spazio per un narratore. In esso, tranne brevissime didascalie, tutti parlano, anche le sedie, creando vortici di parole. E quando si chiude il libro finito e si guarda la copertina con l'immagine dell'uomo, bagnato fradicio, che si protegge a malapena con un ombrello da turbinosi vortici di pioggia e bora, allora, ancora ci si chiede: "Che cosa è?».

1. «Questo viaggio di immissione e fuga da un tunnel senza fine produce l'effetto di una discontinuità, interiore nei comportamenti esterni, che fa di questo nuovo romanzo dello scrittore triestino sicuramente il più inquieto e tormentato all'interno di una esemplare carriera d'autore, come le sue acque del "suo" Danubio avessero subito una tempestosa accelerazione che si manifesta anche nelle pulsazioni aritmiche del linguaggio, della scrittura». (Walter MAURO, Il tempo 03.06.2001).

2. V. TimmeL, Il magico taccuino (versione e coordinamento condotti sui manoscritti inediti originali a cura di Anita Pittoni per desiderio e con l'approvazione dell'Autore), Trieste: Lo Zibaldone, 1973. D'ora in avanti nel testo con la sigla MT seguita dal numero della pagina. Nel corso del testo si farà ricorso alle seguenti opere con le relative sigle seguite dal numero delle pagine: Claudio Magris, La mostra, Milano: Garzanti, 2001 (LM); Claudio MaGris, Fra il Danubio e il mare, ivi, 2001 (DM). 
È un racconto? Un' operetta teatrale? E' un film? Una biografia lampo? Una musica di parole? E'un po' tutte queste cose insieme. Forse, ci viene da pensare, è il ritratto buio e bistrato di un fantasma. Perché non si deve dimenticare che il protagonista, Vito Timmel, è morto. E' un'ombra, anzi l'ombra di un'ombra. Se ne sta «accantucciato» in un angolo del palcoscenico, le braccia incrociate, a vedere "mettere in mostra» la propria vita, uno spettacolo dove compare e parla come personaggio fra i personaggi. Insomma Timmel è contemporaneamente qui e lì: sulla scena e fuori della scena, dentro la vita e fuori della vita. E' sdoppiato sulla scena: vive e si guarda vivere. Ed è inutile dire che è lo sguardo del morto, di chi c'è e non c'è, a coincidere col punto di vista del narratore inesistente che qui dovrebbe essere, invece, anzi è, il regista.

Regista? Sì, perché mai come con quest'opera Magris, che da giovanissimo voleva fare il regista cinematografico (se Giovanni Getto non lo avesse portato a Torino per farne lo studioso eccellente e originale che poi è diventato), ha raggiunto una qualità filmica così prepotente. Solo un film potrebbe tenere il passo con l'accelerazione delle immagini, coi bruschi passaggi di scena del testo; solo un film potrebbe rendere la fantasmagoria mesmerizzante di inquadrature dove si affiancano e si susseguono vertiginosamente luoghi, persone, stelle, mare, piazze, celle manicomiali, primi piani di tele, osterie, fuochi d'artificio e buche di cimitero.

Mi era arrivata voce che il testo - che è stato scritto per essere messo in musica da quello straordinario compositore sperimentale triestino che è Fabio Nieder — avrebbe avuto la messa in scena nientedimeno che di Greenaway (ma, avendo chiesto all'autore, ho ricevuto una smentita...). Si deve pensare, dunque, a cosa potrebbe diventare questo testo, rutilante di immagini di per sé e musicalmente dissonante, se fosse messo in scena con musica e scenografie di quel genere.

Ma ora, davanti a questo testo nudo e crudo, ci chiediamo «che cosa è?». Che cosa è letterariamente? Innanzitutto, si deve dire che il libro appartiene alla serie di quelle opere che io, in Epica sull'acqua, ho chiamato "Biografie imperfette». ${ }^{3}$ La mostra è da mettere vicino a Un altro mare, a Stadelmann, a Il Conde, e in più ha, come Stadelmann e Le voci, una struttura teatrale, ${ }^{4}$ e come Le voci ha per protagonista delle voci, è fatto soltanto di voci. ${ }^{5}$ Ma è anche parente di

\section{Ernestina Pellegrini, Epica sull'acqua, Bergamo: Moretti\&Vitali, 1997.}

4. "Il teatro può essere una risorsa preziosa per i grandi professionisti della parola non teatrale quando il caso o l'ispirazione li metta di fronte a una materia particolarmente densa e incandescente, una materia non del tutto riducibile, per una ragione o per l'altra, alla linearità di un racconto più o meno canonico o a una razionalità di tipo saggistico. Il caso del testo di Magris mi sembra, da questo punto di vista, paradigmatico. Con quale altro linguaggio, in quale altro spazio espressivo l'autore di Danubio e di Lontano da dove avrebbe potuto dare alla vicenda di Vito Timmel una collocazione ambientale e storica così rapsodica e al tempo stesso così corposa, una risonanza allegorica così immediata, una profondità introspettiva (e persino, verrebbe voglia di insinuare, autobiografica) così ineluttabile?» (Giovanni RABONI, Il Corriere della Sera).

5. «I temi di fondo, affidati alla figura contrastata di Timmel, e segnati da un bruciante graffio autobiografico, sono quelli che troviamo variamente nell'opera di Magris: la dissociazione dell'io, la fatalità e la responsabilità del male, il confronto tra vita e letteratura, il disincanto 
certi studi di Magris sull'irrazionalismo di destra, su Hamsun, per esempio, o su Robert Walser (che vagheggia «l'utopia di servire»), o su altri artisti che come Kafka hanno creduto che la salvezza stesse nell'inerzia e nell'apatia, nella regressione e nella schiavitù, nello sparire nell'ombra e nella dipendenza totale. Timmel, a un certo punto, nella Mostra dice: "Xe cussì triste, esser liberi» (LM, 71). E che nel Magico Taccuino - in un punto dove auspicava l'avvento di «esseri risoluti, forti più dell'acciaio, esseri che regolino, fondino, inquadrino le masse», delle «esistenze-comandi» che riescano ad affliggere i ribelli, ad essere, diceva, addirittura dei «carnefici» (MT, 21) - masochisticamente aveva scritto: «Bisogna assolutamente dipendere» (MT, 21).

Basaglia, nella sua introduzione, dice che per Timmel «forse la malattia è l'identificazione totale nelle cose, da cui non può più distanziarsi per esserne interprete o portavoce. E' l'annullamento della distanza fra l'io e le cose in cui l'io si disperde. Ma in questo processo di autoeliminazione - si chiede quanto c'è di intenzionale, voluto e quanto è stato prodotto? (MT, XXXII).

$\mathrm{E}$ a questo punto voglio fare una parentesi che vorrebbe funzionare anche come domanda indiretta all'autore. Nel recentissimo saggio su se stesso prima ricordato, Fra il Danubio e il mare, lo scrittore dice che quel racconto in forma teatrale sarebbe stato impensabile «senza la grande frattura avvenuta alcuni anni fa nella [sua] esistenza e senza il buio, doloroso e anche melmoso, attraversato in quegli anni, che pervade il testo» (DM, 40). L'autore rivela, dunque, un sottofondo autobiografico (il dolore per la perdita della "sposa-luce», per esempio) e lascia pensare che il testo sia stato ideato negli ultimi anni. Magris dice anche che "La mostra squarcia e allarga ferite che Danubio e Microcosmi avevano, nonostante tutto, ricomposto» (DM, 42). Ma io ho una copia del Magico taccuino di Timmel, che Magris mi ha regalato nel 1993, che è tutta postillata da commenti a lapis interessantissimi che mi fanno pensare che l'ideazione di lavorare su questa stralunata figura di artista risalgano a tanti anni prima, certo a tempi anteriori al 1993. E allora chiedo: a quando risale l'ideazione di questa biografia lampo di un uomo che ha "peccato per difetto", di questo "superuomo-sottouomo» che ha peccato per una sorta di omissione nei confronti della vita? Questo testo è o non è un punto d'arrivo della sua scrittura?

È — come vuole Lorenzo Mondo su «La Stampa» del 24 maggio 2001 «uno spartiacque, un punto di non ritorno nella scrittura di Magris», quasi il raggiungimento di un nuovo registro, per cui - come ha scritto De Michelis - Magris «saggista e narratore, dopo questo libro è soprattutto poeta, abbacinante poeta del dolore»? O piuttosto siamo portati a pensare - come credo io - che questo testo lungamente incubato su una delle tante figure di «superuomini alla rovescia», di superuomini per sottrazione - che ha per fratelli Enrico Mreule di Un altro mare, il pescatore di cadaveri de Il Conde, il don-

sempre incalzato da una speranza più alta e sfuggente. Con in più, la sensazione vaga di uno spartiacque, di un punto di non ritorno nella scrittura di Magris». (Lorenzo MONDO, La Stampa, 24-5-2001). 
giovanni platonico delle Voci, Krasnow della Sciabola, il servo di Goethe che si suicida nella lavanderia di un ospizio in Stadelmann - rappresenti un momento liberatorio, di disintossicazione del dolore che apre la strada non alla poesia ma a un modo nuovo, largo e arioso del raccontare? Non è un caso che questa storia di «addio alla vita» trovi la sua concreta realizzazione, la sua musica strappata, la sua "stesura selvaggia» — come la chiama Magris — in questi ultimi e dolorosi anni, in concomitanza con tante pagine ariose di Utopia e disincanto. Penso che quel processo di "guardare in faccia la tenebra» (DM, 42) significhi anche una definitiva disintossicazione dal proprio «materialismo infelice», che stia, insomma, per lasciare il campo a prossime storie magari più avventurose, più vitalisticamente salgariane.

La Mostra è un testo violento e visionario, senza sponde di genere, che mette in scena, in una tragicomica sintesi, il destino minimo, semicancellato di un uomo che ha scelto di rifiutare caparbiamente il mondo. Il protagonista - l'ho detto - è un'ombra. A quasi trent'anni dall'uscita del Magico taccuino, di quel "protervo e puerile vangelo di se stesso" (MT,XX), Magris ci dà questo Requiem, che ne è lo specchio e la continuazione, la radiografia esplicativa e la sublimazione. La mostra è un diaro scritto su un diario, è il sogno di un sogno di un altro. Si srotolano uno dopo l'altro, in apparente disordine, le vicende di un'esperienza-limite e dei micidiali meccanismi dell'esclusione sociale a cui è stata sottoposta. Vediamo i fotogrammi slegati di una fine che non finisce mai. Grandi campiture pittoriche in cui perdersi, struggenti sfondi melodici in cui perdersi, l'eco sonora di una vita senza centro che rimbomba nello spazio cosmico allontanandosi fino a scomparire. Basti citare la fine del racconto. Sono le ultime parole di Timmel: "che scalmana, tutti si agitano, per niente, scordata avventura - sono passati anni luce, giro con l'asse terrestre intorno al sole, astri sorgono e tramontano, i colori dell'alba e della sera, il sole, i soli sui muri $[\ldots] »(\mathrm{LM}, 74)$.

Non c'è trama. Il plot è una sapiente sfilacciatura per una trama che è stata raccontata altrove. Un concerto di voci e di lingue (dialetto triestino, tedesco, latino, un italiano arcaico, filastrocche, cantilene, spezzoni di versi sublimi, citazioni da Baudelaire, gridi, lagne, parole tuttattaccate, borbottamenti e cori borborigmi). Chi è il protagonista? La vera protagonista è la lingua, una musica «scordata» (scordata come la «scordata avventura»). Molte parole hanno significati multipli, indistinti. Pensate a certi aggettivi come «randagio», "cedevole», a certe parole come "mostra», "mostro». Sono dei veri e propri bonsai linguistici. Eppure, in mezzo a questa Babele concentrazionaria, nello spazio stretto del palcoscenico (cella di un ospedale psichiatrico, sala di un museo) la parola resta un evento meraviglioso e pericoloso, quasi che la parola teatrale conservasse in sé la silenziosa impassibilità degli dei e accogliesse riscattandolo il rimuginio di questa tragedia senza eroe, di questo linguaggio senza più soggetto.

Al centro della rappresentazione - questo mi sembra importante notarlo - di concreto c'è solo la lingua, con i suoi soprassalti, i suoi strappi, le sue impennate liriche e le sue cadute scurrili. Il resto è solo un immane, minac- 
cioso, crudele, trascolorante sogno. Personaggi larvali appaiono e scompaiono, con le loro ossessioni, i loro sogni e i loro spaventi. Le sedie cantano in coro, le stelle fanno da uscio al cielo, le osterie sono affrescate come chiese. Tutto è irreale, svaporato, dolorosamente impalpabile. L'irrealtà, sia memoria semicancellata o delirio, fa da padrona in questo caleidoscopio di parole surriscaldate. In questo libro c'è il succo di una vita da ascoltare, una vita di arte e di follia, ma c'è anche la sua esibizione fossile. Perché non si deve dimenticare che al centro c'è anche l'episodio dell'allestimento di una mostra postuma dei quadri dell'artista all'ospedale psichiatrico di Trieste, una mostra sulla quale sovrasta la voce metallica e pomposa del Direttore che sciorina le sue diagnosi cliniche. E fra questi due piani - quello della vita passata che risuona sbandata e randagia, inafferrabile trascorsa sciupata, davanti agli occhi di quel cedevolissimo fantasma "incattucciato», e quello della mostra celebrativa c'è un continuo stridìo, un violento cortocircuito. Un cortocircuito fra ciò che passa e ciò che rimane. E allora sotto processo in questo libro - vien da pensare - finisce la memoria, e i meccanismi che regolano i rapporti fra presenza e assenza.

La mostra. Perché questo titolo? Perché l'accento batte su quel gesto profanatore del mettere in mostra, in vetrina, in questo caso in un ospedale psichiatrico, i frutti di una immaginazione eccitata e franante; mette l'accento su quell'esibizione profanatrice-riparatrice che sottopone alla vista e al giudizio degli altri il succo segreto di una vita, la vita di chi - e cito dal Magico taccuino (sono parole di Timmel) — si descriveva «chiuso, ozioso, disinteressato", e giudicava il fare l'amore come un "caotico festival» (MT, 19). Così come giudicava l'uomo «una muffa preistorica» (MT, 20) e la morte «un foro ombroso» (MT, 13). Un artista che nel suo quadro intitolato Gli anonimi aveva dipinto dei corpi nudi distesi su degli scogli in un paesaggio infernale e apocalittico, con trombe d'aria e un mare fumigante, corpi nudi e ammassati, scomposti, che alzano tutti, chi con forza chi mollemente, un braccio al cielo (come chi a scuola venendo chiamato è obbligato a dire "presente", "eccomi qua», "ci sono"; e qui risponde a un'atmosfera da Giudizio Universale).

Allora, dicevo, perché La mostra? Se non sembrasse un gioco banale di parole direi che si tratta della "mostra di un mostro». C'è una definizione curiosa di Benedetto Varchi in un dizionario etimologico per mostro: "mostri si chiamano ogni volta che hanno o più membra o manco membra, o membra non proporzionate e convenevoli. Quasi mostri si chiamano le femmine, dice Aristotile, benché nel vero sono mostri necessari». Non è una citazione bizzarra che non c'entra nulla. Potrebbe entrarci se si pensa a quella duplice, contraddittoria immagine di Timmel con le braccia incrociate, quindi come se fosse senza braccia, chiuso in sé, chiuso al mondo, e di Timmel che diventa stella a cinque punte (sempre esagerato, comunque, sempre mostro); e se si pensa alla sua regale misoginia, alle sue donne angelo e alle sue donne diavolo, ai suoi "mostri necessari» A mio parere, le pagine dedicate alla donna sono fra le più belle e intense. Ve ne cito una: 
Mia Alcesti, stella del Settentrione, splendore nella notte scesa e sparita nelle tenebre, il tuo riso e sorriso candide spume inghiottite dalla grande cloaca, laggiù, per me, e io, con le lacrime che mi scendevano sul viso insozzandosi di fetido sudore, felice di non scendere, di restare a galla poggiando su di te, spingendoti sotto nelle acque limacciose, avido di respirare sul tuo soffocare - eppure straziato e sincero, sì, perché sapevo che non c'era più niente senza di te, grande scudo che proteggeva dal fuoco la mia nave marcia arenata sotto le mura di Ilio. Solo in te rinascevo ogni volta e ormai invece ero là sotto anch'io, sputacchio assorbito dalla terra della fossa - Non guardarmi, mi hai detto, non sono Euridice, non sono qui perché tu scenda nell'Averno, ma scendo io perché tu non ne conosca l'orrido niente.

Mia Alcesti, amaro ritorno, odioso spettacolo di stanze vuote

$\mathrm{O}$ reggia, come posso entrare in te, abitarti?

La sorte è cambiata, tutto è mutato sono perduto [...]

[...] Come fu brava, l'altra, a domesticarmi alle erotiche arguzie [...] tumulto dell'amplesso, inspiegabile, aria viziata, meandri infiniti della volgarità, filtri di dominio e schiavitù mescolati nella saliva che succhiavo con baci feroci, umiliati - li preparava in storte e alambicchi, il demone glieli suggeriva nelle notti stillanti di sperma e di sudore [...] (LM, 57-8).

Perché, dunque, La mostra? Perché perduta è ogni profondità, e tutto è mostrato con violenza, nella violenza inattiva del dialogo, nella tirannia esercitata dal dialogo, in un'opera che è anche - come negarlo? - un ininterrotto monologo. Basaglia diceva, infatti, che Timmel «riduce il messaggio a un monologo senza interlocutore» (MT, XXXIII). Protagonista è il malinconicissimo Timmel, fuori di testa, viennese triestino viandante senza patria, ma protagonisti sono anche gli altri: il clan dei suoi amici e nemici, i suoi carcerieri e i suoi volenterosi guaritori, i suoi quadri giganti e i suoi dipinti a misura di francobollo, le sue smanie di solitudine e i suoi raptus erotici o assassini, i brandelli delle sue sgangherate memorie e i commenti di coloro che lo conobbero, amandolo, sfruttandolo, canzonandolo (come quell'ingegnere collezionista dei suoi quadri che lo costringe a bere un bicchiere d'orina). Protagonisti sono anche il Direttore, gli infermieri, un pittore macabro traduttore di Baudelaire, gli amici, le sedie, i pazzi e i sani, il regno della vita e il regno della morte (ci si chiede: chi taglia i confini?).

Perché La mostra? Perché la vita di Timmel è racchiusa e sperperata anche in questi dialoghi che non sono un dialogo, dove ognuno parla per conto suo. Chiacchiere eroiche. Perché seppure è evidente al lettore inchiodato al macabro, tenerissimo spettacolo, di essere stato scaraventato in quell'antimondo che è l'immaginario, qualcosa gli suggerisce che tutto ciò implichi anche un enigma. Ci si chiede: chi pensa chi? Ci si trova in uno spazio multiplo, si è investiti da una parola plurale. Tutto è mostrato, e non esiste nulla di implicito, di segreto, di difeso dalla violenza, dalla manipolazione, dall'irregimentazione, dalla volgarità del mondo e del potere. Cos'è la libertà? Cos'è veramente privato o sacro? Domande gigantesche o puerili, forse tutte e due le cose insieme. 
La morte di Timmel con cui si apre lo spettacolo è infinitizzata, resa inconcludente, nella volontà collettiva di una eternità immediata, quella della mostra, quando la costruzione della memoria e le sue strategie sembrano solo il feticismo sentimentale di una spuria sopravvivenza, il frutto di un addomesticamento e di una riparazione sociale, la colonizzazione sociale dello spaventoso nulla, quel nulla dove Timmel invece vuole andare a finire:

La tromba del Giudizio [...] suona, suona [...] mi sveglio di colpo e perdo l'equilibrio, cado, non finisco più di cadere, giugiugiù, è meraviglioso sono un punto, roteo con dugentilioni di stelle, se apro le braccia e le gambe, ecco, sono una stella, una stella a cinque punte [...] tanti punti luminosi, tanti tanti, petali di un sorriso, una margherita si sfoglia nella notte. Tutti i disagi si placano. Dio è guarito, niente più raffeddore, e quello là cade, cade dal muretto felice, mi pare di conoscerlo ma non so come si chiama, no, von Thummel no, e neanche $[\ldots]$ ecco, è sparito, come dev'essere felice $[\ldots]$ (LM, 73).

Magris combacia e non combacia col proprio personaggio, in questa biografia scritta dall'interno, scritta da due punti di vista opposti: della vita e della non-vita. La poesia scatta dallo scontro e dall'impossibilità di scegliere da che parte stiano le ragioni, se in quelle della pienezza smemorata o in quelle dello inevitabile svuotamento. Per questo all'inizio del racconto si sovrappongono i rumori dei botti dei fuochi d'artificio e dei tappi delle bottiglie di champagne con i tonfi delle palate di terra su una bara.

Si mette in scena, letteralmente, la fine di un uomo, ma anche se ne tenta la resurrezione impossibile, col suo restauro biografico. L'autore gli presta anche le proprie emozioni più travolgenti e segrete, tentando la ricostruzione di una identità che si era volatizzata in tutto: nel nome, nei significati, nelle relazioni sentimentali e sintattiche. Resta una biografia laconica e ricostruita a brandelli attraverso le parole di Timmel, con stralci del Magico taccuino, e attraverso le parole di altri personaggi, primo fra tutti il pittore macabro Cesare Sofianopulo, suo vero doppio (fatto sta che in un punto Timmel e Sofianopulo parlano insieme e dicono la stessa cosa), ma anche attraverso cori di pazzi, cori di oggetti, voci dal passato, scrosci di pioggia. E' nato così un libro che «restituisce — ha scritto Giampaolo Rugarli — il brusio della strada».

È nato un requiem, cioè una forma di preghiera, di pietosa lamentazione funebre che è anche, sotto certi aspetti, una profanazione biografica, cioè la messa in mostra di una vita che ha fatto di tutto per essere dimenticata. Si tenta di fare, fra pietas e accanimento conoscitivo, lo squadernamento di una identità che ha voluto, almeno da un certo punto in poi della propria vita, soltanto "desmentegar», dimenticare. Si capisce tutto ciò nell'ultima scena di questo racconto che non ha un "prima» e un "poi», la scena in cui si vede arrivare un automezzo della nettezza urbana e Timmel scambia il suono del clacson con la tromba del Giudizio Universale (e noi si pensa: immondizie, bilancio 
di una vita - come sono vicini i confini), e a lui sembra di cadere, di non finire mai di cadere e si sente di essere una stella a cinque punte o una margherita da sfogliare, mentre canta: «Sì dimenticar / perfino gli occhi tuoi / scordar che tu mi vuoi / scordar che voglio te [...]» (p. 73).

Vito Timmel cerca di dimenticare la vita, di dimenticare e di ottundere la propria sofferenza - come scrive Magris - «in una regale, apatica e anarchica autodistruzione» (DM, 40). «Ne è uscito un piccolo capolavoro, centro di tensioni enormi» — come ha scritto Luca Doninelli su Il Giorno: «Magris, autore di centinaia di pagine impeccabili, avverte sempre più, anno dopo anno, il bisogno di sacrificare ogni impeccabilità affinché il dono di sé sia totale. E la totalità di un uomo è sempre peccabile. Questo fa di lui l'unico vero maestro, forse, della nostra cultura».

A questo punto ci si chiede che tipo di lettura chieda un testo come $L a$ mostra? Una lettura eternizzante o una lettura storica? È un testo della disperazione esistenziale e del pessimismo metafisico, o piuttosto - come già aveva notato Lukàcs per il Woyseck di Buchner - questa lettura ridurrebbe la carica storica e politica socialmente eversiva e pericolosa del messaggio di un individuo e di un popolo straziati da un progresso che non è il loro? Timmel come Woyseck - la cui traduzione è stata per Magris un vero e proprio «giro di boa»- è uno dei tanti "volti anonimi e oscuri» che si succedono, "forse invano sulla scena del mondo». ${ }^{6}$

Viene da pensare, per concludere, che La mostra sia la storia di una doppia mise à mort. Come in Psycho, il film di Hitchcock, abbiamo la combinazione impossibile e contraddittoria fra la necessità di uccidere definitivamente il passato e quella di farlo continuare a vivere. Tutto è presente, reversibile e sacrificato. E' questo, credo, il nodo profondo, irriducibile di questo libro.

In una prosa spezzata e imprevedibile, vertiginosamente «bassa» e poeticamente strampalata si susseguono sulla scena balbettii, gridi, cori di sedie e semicori di pazzi, paradisi veri e locande paradiso, spose celestiali e spose diavolo, infiniti soffittati di stelle e cappelle sistine giocattolo, fuochi d'artificio e casse da morto. Tutto è come deportato in uno spazio-tempo, quello della mostra, dove rintocca inesorabile il gong di una condanna. Requiem o condanna - ed è lo stesso - per un uomo che "piangeva, anche, ma così, giusto per piangere» (LM, 22).

$\mathrm{E}$ a questo punto, in coda, qualche riflessione generale e qualche citazione sul concetto di frontiera nell'opera di Claudio Magris. Un tema, questo, onnipresente, che ha trovato in La mostra la sua più completa ed esplicita realizzazione rappresentativa, ma che era presente sì dal primo testo narrativo, Illazioni su una sciabola, in cui Don Mario, che si ritiene ormai «un pensionato dello spirito», sogna di «andare dall'altra parte», perché il mondo, "come un vestito striminzito» comincia a stargli stretto:

6. G. Buchner, Woyzeck, traduzione di C.Magris, a cura di H. Dorowin, Venezia: Marsilio, 1988 (Nota del traduttore, p. 11-23). 
Ormai il mondo mi sta stretto come un vestito striminzito; tutto intorno a me è un limite, anche quel blu del mare e il rosso di certe sere sull'orizzonte di quel mare: limiti incantevoli che ho tanto amato fin da ragazzo e amo ancora, e di cui il Signore, ma limiti anch'essi. E io mi sento stanco, vorrei uscire andare dall'altra parte ${ }^{7}$

Ma troviamo una delle definizioni più chiare e quasi teoriche del concetto di frontiera nel volume scritto a quattro mani con Angelo Ara, Trieste. Un'identità di frontiera del 1982, in cui si tenta di definire una vera e propria psicologia del «cittadino del confine»:

Se Trieste è una frontiera, quest' ultima diviene, in alcune opere letterarie, un modo di vivere e di sentire, una struttura psicologica e poetica. La frontiera è una striscia che divide e collega, un taglio aspro come una ferita che stenta a rimarginarsi, una zona di nessuno, un territorio misto, i cui abitanti sentono spesso di non appartenere veramente ad alcuna patria ben definita a almeno di non appartenerle con quella ovvia certezza con la quale ci si identifica, di solito, col proprio paese. Il figlio di una terra di confine sente talora incerta la propria nazionalità oppure la vive con una passione che i suoi connazionali stentano a capire, sicché egli, deluso nel suo amore che non gli sembra mai abbastanza corrisposto, finisce per considerarsi il vero e legittimo rappresentante della sua nazione, più di coloro per i quali essa è un dato pacificamente acquisito.

Ma la frontiera, la quale separa e spesso rende nemiche le genti che si mescolano e si scontrano sulla sua linea invisibile, anche unisce quelle stesse genti, che si riconoscono talora affini e vicine proprio in quel loro comune destino che grandi madrepatrie non riescono a capire - in quel loro sentimento segreto di inappartenenza e in quell'indefinibilità della loro identità. ${ }^{8}$

Magris riflette ossessivamente nei suoi scritti, saggistici e narrativi, sul motivo della frontiera. Sarebbe impossibile procedere a una puntuale catalogazione. Si può solo fare un cenno a quelle occasioni in cui il tema compare in una chiave più strettamente autobiografica. Come è successo in una intervista radiofonica dal titolo Paesaggio con figure, del 14 febbraio 1993, in cui lo scrittore tornava ai primi ricordi infantili della "cortina di ferro»:

[...] la frontiera correva pochissimi chilometri da casa mia, la vedevo quando andavo sul Carso a fare passeggiate: era la "cortina di ferro», quella che divideva il mondo in due parti. Era un'esperienza curiosa e duplice; oltre a quella frontiera cominciava un mondo che da una parte era sconosciuto, minaccioso, terribile - il mondo dell'impero di Stalin, almeno sino a quando, negli anni '50, dopo la rottura fra Tito e Mosca e con la progressiva normalizzazione dei rapporti fra Italia e Jugoslavia, quella frontiera ha cominciato ad aprirsi. Ma quei luoghi erano contemporaneamente familiari, perché erano i luoghi che 
avevo conosciuto da bambino, dato che erano, appunto, le terre che avevano fatto parte dell'Italia, ed erano poi passate alla Jugoslavia alla fine della seconda guerra mondiale.

Quindi, io avevo il sentimento, naturalmente molto inconsapevole, che avrei dovuto valicare in qualche modo - non solo materialmente, ma anche spiritualmente - quella frontiera, rimpossessarmi di quel mondo che era anche mio. Di qui il senso della frontiera, della difficoltà e necessità di varcare le frontiere - e non parlo solo delle frontiere nazionali, statali o politiche, ma anche psicologiche, culturali, d'ogni genere.

Queste considerazioni riguardo alla necessità e all'inevitabilità dell'attraversamento delle frontiere, che investe il processo stesso del vivere, del crescere e del morire, è al centro di un bellissimo saggio, non molto conosciuto, dal titolo Chi è dall'altra parte? Considerazioni di frontiera, uscito sulla «Nuova Antologia» dell'aprile-giugno 1992:

I confini vengono spostati, spariscono e improvvisamnte ricompaiono: con essi si trasforma in maniera errabonda il concetto di ciò che chiamiamo Heimat, patria. Città ed individui si trovano spesso ad essere degli «ex» e quest'esperienza dello spostamento, della perdita del mondo, non riguarda solo la geografia politica, ma la vita in generale. Il mio Stadelmann dice che ognuno è un ex di qualcosa, anche se non sa di esserlo. ${ }^{10}$

9. Claudio Magris, Paesaggio con figure, intervista in diretta alla Radio del 14 febbraio 1993. Esiste una copia dattiloscritta da sbobinatura nell'archivio personale dello scrittore a Trieste.

10. Claudio MAGRIS, "Chi è dall'altra parte? Considerazioni di frontiera", Nuova Antologia, aprile-giugno 1992, p. 50-61. Riprende alcuni temi svolti alla Berliner Lektio del 1 settembre 1991. Al Carrefour Littéraire di Strasburgo il 9 novembre 1991; alla Freund-Gesellschaft di Vienna nell'aprile 1992 e all'Università di Salizburg nel luglio 1992. 